\title{
Physiological Changes During Prone Positioning in COVID-19 Acute Respiratory Distress Syndrome
}

\section{Dear Editor,}

Prone positioning is an established treatment modality in acute respiratory distress syndrome (ARDS) and has been employed in the management of ARDS in severe 2019 novel coronavirus disease (COVID-19) in the intensive care unit (ICU) with varying clinical and physiological responses. ${ }^{1,2}$ To date, Singapore has seen 44,122 COVID-19 patients, with the majority of inpatients managed at National Centre of Infectious Diseases (NCID). NCID ICU has managed 36 mechanically intubated patients thus far. We retrospectively examine the first 20 patients in NCID ICU and describe our early experience of prone positioning in COVID-19 ARDS including identifying potential early physiological indicators of poor response to prone positioning.

\section{Method}

We retrospectively reviewed the medical records of all individuals with COVID-19 ARDS who received prone positioning at the NCID from 8 February to 29 March 2020. Since the first reported case of COVID-19 in Singapore on 23 January 2020 to the time of writing, NCID ICU has managed 20 mechanically intubated patients. Baseline clinical characteristics and important physiological indicators during the first prone positioning episode were examined. Physiological indices at various time points were compared using the Wilcoxon matched-pair signed rank tests. $P$-value of $<0.05$ was considered statistically significant. Ethical and institutional board review were waived due to provision under the Infectious Diseases Act, Singapore.

\section{Results}

Seven out of 20 cases received prone ventilation; majority were male $(86 \%)$ with a median (interquartile range [IQR]) age of 62 (39-73) years. All patients received invasive mechanical ventilation for hypoxemic respiratory failure and the cases fulfilled the criteria for moderate-to-severe ARDS with evidence of ventilatory insufficiency (Table 1). Radiological imaging revealed that all cases consistently had bilateral pulmonary infiltrates and consolidation worse in the bases and dependent areas. The patients received lung protective ventilation with applied tidal volume of $6.0-7.5 \mathrm{~mL} /$ kg ideal body weight. Prone positioning was employed within 72 hours of endotracheal intubation in 5 cases (Day 7 and 8 for the remaining 2 cases). There was a significant improvement in oxygenation following prone positioning as early as 2 hours into prone positioning (Fig. 1). $\mathrm{PaO}_{2}: \mathrm{FiO}_{2}$ (partial pressure of arterial oxygen: percentage of inspired oxygen) ratio was $99(86-111) \mathrm{mmHg}$ at baseline and $180(162-226) \mathrm{mmHg}$ after prone positioning $(P=0.02)$. Of note, $\mathrm{PaO}_{2}: \mathrm{FiO}_{2}$ ratio decreased at post prone period when compared to values at 16 hours of prone positioning $(P=0.04)$, suggesting that some of the physiological benefit was not sustainable when supine position was resumed (Fig. 1). Multiple prone sessions were often required at a median of 3 (1-9) sessions per patient. The median (IQR) duration of prone ventilation was $16.2(15.1-17.5)$ hours.

Arterial partial pressure of carbon dioxide $\left(\mathrm{PaCO}_{2}\right)$ trend during prone positioning is shown in Figure 2. There was a decrease in $\mathrm{PaCO}_{2}$ at 2 hours into prone positioning in 5 out of 7 patients but significant statistical reduction was only observed at 16 hours into prone positioning when compared to baseline data $(P=0.04)$. Upon returning to supine position, $\mathrm{PaCO}_{2}$ returned to baseline level (Fig. 2). Dynamic respiratory compliance did not improve post prone positioning (25 [21-27] versus 27 [21-32] $\mathrm{mL} / \mathrm{cmH}_{2} \mathrm{O}, P=0.67$ ). Some patients received adjunct therapy: vasopressors $(n=4)$, renal replacement therapy $(n=2)$, glucocorticoid therapy $(\mathrm{n}=3)$ and broad-spectrum antibiotics cover $(\mathrm{n}=7)$. All patients received neuromuscular blocking agent during prone sessions. We did not observe any significant adverse events. One patient had recurrent regurgitation that was managed with prokinetics (Table 1). 
Table 1: Patient Characteristics and Outcomes

\begin{tabular}{lcccccccc}
\hline Case & $\mathbf{1}$ & $\mathbf{2}$ & $\mathbf{3}$ & $\mathbf{4}$ & $\mathbf{5}$ & $\mathbf{6}$ & $\mathbf{7}$ \\
\hline General characteristics & & & & & & & & \\
\hline Age, years & 73 & 39 & 75 & 54 & 36 & 64 & 62 \\
Sex & Male & Male & Female & Male & Male & Male & Male \\
Body mass index, $\mathrm{kg} / \mathrm{m}^{2}$ & 14.2 & 27.6 & 19.6 & 24.7 & 25.4 & 26.6 & 40.2 \\
APACHE score & 7 & 10 & 16 & 9 & 22 & 20 & 32 \\
\hline
\end{tabular}

\section{Medical therapy}

Vasopressors - no.
Neuromuscular blockage
Renal replacement therapy
Glucocorticoid therapy

Ventilator setting before prone ventilation

\begin{tabular}{|c|c|c|c|c|c|c|c|}
\hline Set volume, ml per kg IBW & 7.0 & 7.5 & 7.3 & 7.1 & 6.0 & 7.0 & 6.3 \\
\hline $\mathrm{FiO}_{2}$ & 1.00 & 1.00 & 0.70 & 0.60 & 1.00 & 0.95 & 0.5 \\
\hline PEEP, $\mathrm{cmH}_{2} \mathrm{O}$ & 12 & 14 & 10 & 12 & 14 & 15 & 14 \\
\hline \multicolumn{8}{|c|}{$\begin{array}{l}\text { Respiratory system mechanics before prone } \\
\text { ventilation }\end{array}$} \\
\hline Plateau pressure, $\mathrm{cmH}_{2} \mathrm{O}$ & 26 & 26 & 24 & 27 & 22 & 28 & 24 \\
\hline Static compliance, $\mathrm{mL} / \mathrm{cmH}_{2} \mathrm{O}$ & 32 & 32 & 25 & 32 & 56 & 35 & 38 \\
\hline
\end{tabular}

Arterial blood gas before prone ventilation

\begin{tabular}{|c|c|c|c|c|c|c|c|}
\hline $\mathrm{PaO}_{2}, \mathrm{mmHg}$ & 97 & 99 & 73 & 67 & 86 & 81 & 63 \\
\hline $\mathrm{PaO}_{2}: \mathrm{FiO}_{2}, \mathrm{mmHg}$ & 97 & 99 & 104 & 111 & 86 & 81 & 126 \\
\hline $\mathrm{PaCO}_{2}, \mathrm{mmHg}$ & 66 & 69 & 61 & 49 & 77 & 70 & 47 \\
\hline Arterial $\mathrm{pH}$ & 7.24 & 7.24 & 7.15 & 7.41 & 7.28 & 7.14 & 7.36 \\
\hline \multicolumn{8}{|l|}{ Prone positioning } \\
\hline Average duration per session (h) & 15.5 & 16.0 & 18.0 & 14 & 13.7 & 17.3 & 17.5 \\
\hline \multicolumn{8}{|l|}{ Adverse events } \\
\hline Haemodynamic instability & - & + & - & - & - & - & - \\
\hline Other & - & + , Vomit & - & - & - & - & - \\
\hline $\mathrm{PaO}_{2}: \mathrm{FiO}_{2}, \mathrm{mmHg}$ & 180 & 183 & 165 & 184 & 267.5 & 116 & 226 \\
\hline $\mathrm{PaCO}_{2}, \mathrm{mmHg}$ & 50 & 61 & 54 & 58 & 56 & 61 & 57 \\
\hline \multicolumn{8}{|l|}{ Outcome } \\
\hline Tracheostomy & & + & - & - & - & - & - \\
\hline Length of ICU stay, days & 98 & 68 & 27 & 23 & 8 & 8 & 45 \\
\hline Extracorporeal membrane oxygenation & - & - & - & - & - & + & - \\
\hline Survival status & Survive & Survive & Demise & Survive & Survive & Demise & Survive \\
\hline
\end{tabular}

$\mathrm{PaCO}_{2}$ : Arterial partial pressure of carbon dioxide; $\mathrm{PaO}_{2}$ : Arterial partial pressure of oxygen; FiO 2 : Fraction of inspired oxygen; IBW: Ideal body weight; ICU: Intensive care unit; PEEP: Positive end-expiratory pressure. '+'=yes/present. '-'=no/absent 
One patient with pre-existing asthma and ischemic heart disease had persistently low $\mathrm{PaO} 2 \mathrm{FiO} 2$ ratio $(<150 \mathrm{mmHg})$ and elevated $\mathrm{PaCO} 2(>60 \mathrm{mmHg})$ despite prone positioning. There was no improvement despite 2 additional prone sessions before he received extracorporeal membrane oxygenation (ECMO) support. We observed 2 mortalities: Case 3 passed away from progressively worsening multi-organ failure while Case 6 developed intracranial bleeding complications following ECMO. The remaining patients survived the ICU stay (Table 1).

\section{Discussion}

We found that prone positioning is a safe and effective management strategy for severe COVID-19 patients with ARDS. Following prone positioning, majority demonstrated almost immediate oxygenation and ventilation improvement. Of note, one individual markedly improved after 1 prone session and was liberated from mechanical ventilation within 14 days. For the rest of the cases, prolonged ICU stay was expected with repeated prone sessions required due to lack of sustained benefit in oxygenation. We did not observe statistically significant changes in respiratory compliance or $\mathrm{PaCO}_{2}$ following prone positioning. This suggests that the improvement in oxygenation was most likely the result of regional improvement in ventilation-perfusion matching and redistribution of blood flow to less diseased parenchyma with resultant decrease in physiological shunt. Interestingly, a few patients demonstrated transient improvement in $\mathrm{PaCO}_{2}$ during prone positioning, which resulted in improvement in arterial $\mathrm{pH}$ and consequently, improved hemodynamic parameters, allowing for a reduction in vasopressor use. It was plausible that the reduction in vasopressor dose contributed to less pulmonary vasoconstriction and the resultant improved pulmonary blood flow may have a role in better oxygenation. The varied trajectory of illness and physiological response to prone positioning suggests that COVID-19 ARDS is clinically heterogenous.

In a busy outbreak ICU with many patients who may need daily prone sessions, judicious use of staff and equipment is essential to optimise manpower and minimise fatigue. We had a minimum of 3 staff to turn each patient prone in a safe and controlled manner. The respiratory therapist or doctor at the head end holds the endotracheal tube and directs the prone positioning while 2 nurses wrap the patient up with bedsheets before turning the patient prone. All staff are in tier 2 personal protective equipment consisting of N95 face masks, goggles or face shield, cap, gown covering up to wrists and gloves. Sedation and drugs are continued and lines untangled prior to turning. Pillows are used to support the chest and pelvis. Staff are in constant communication during the process to ensure safety of the patient. There was no circuit disconnection or tubes and lines dislodgement in our experience. To prevent pressure sores, we use gel pads (e.g. 'occiput donuts') over important pressure points and employ regular lateral shifts in head, shoulders, arms and pelvis.

Our observations suggest that repeated prone positioning (in addition to lung protective ventilation and judicious fluid therapy) is useful for refractory hypoxemia in COVID-19 ARDS in the majority of cases. ${ }^{2,4}$ However, failure to recognise a lack of physiological response to prone positioning may cause the patient to miss the window of opportunity for ECMO, especially in centres which are not ECMO capable and significant lead time is required for ECMO activation. In addition, ECMO is a huge undertaking with many considerations hence requires a concerted multidisciplinary effort that should start with early discussion..$^{5,6}$ In our single patient who received ECMO, the physiological response (or lack of) was clearly distinguishable even from the first prone positioning (Fig. 1 and 2 solid triangle) session and this finding did not considerably change for the subsequent prone session.

In conclusion, we observed marked clinical improvement in COVID-19 ARDS who received early and repeated prone positioning with minimal adverse events. In many cases, lung protective ventilation, prone positioning and best supportive therapy was adequate for management of COVID-19 moderate-to-severe ARDS. ${ }^{4}$ We would like to hypothesise that $\mathrm{PaO} 2: \mathrm{FiO} 2<150 \mathrm{mmHg}$ and $\mathrm{PaCO} 2>60 \mathrm{mmHg}$ despite prone positioning be used as an early marker for poor responders, which should prompt closer monitoring and early consult with an ECMO team. ${ }^{6}$ Our study is limited by the relatively small number of patients and therefore the findings and recommendations should be interpreted in consideration of the healthcare resource and services available at individual hospitals. Our finding calls for further studies to validate the proposed threshold and identification of treatment response subtypes. 


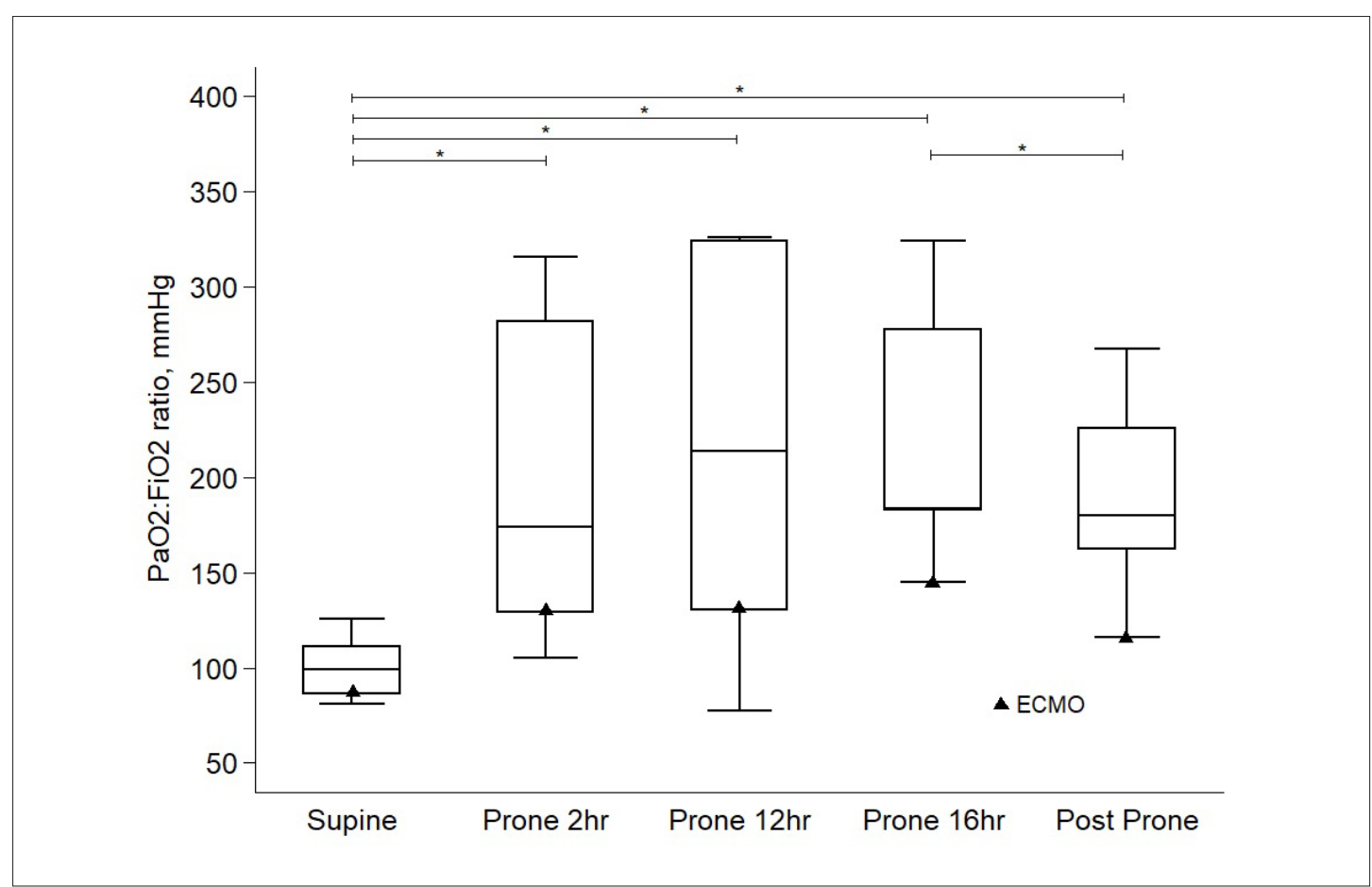

Figure 1: Arterial partial pressure of oxygen:fraction of inspired oxygen ratio (PaO2:FiO2) before, during and after prone positioning in patients with COVID-19 acute respiratory distress syndrome.

Triangle sign denotes the patient who did not response to prone positioning and subsequently required extra-corporeal membrane oxygenation therapy. ${ }^{*} P<0.05$ by Wilcoxon matched-pairs signed-rank test. The rest of between group comparisons were not statistically significant.

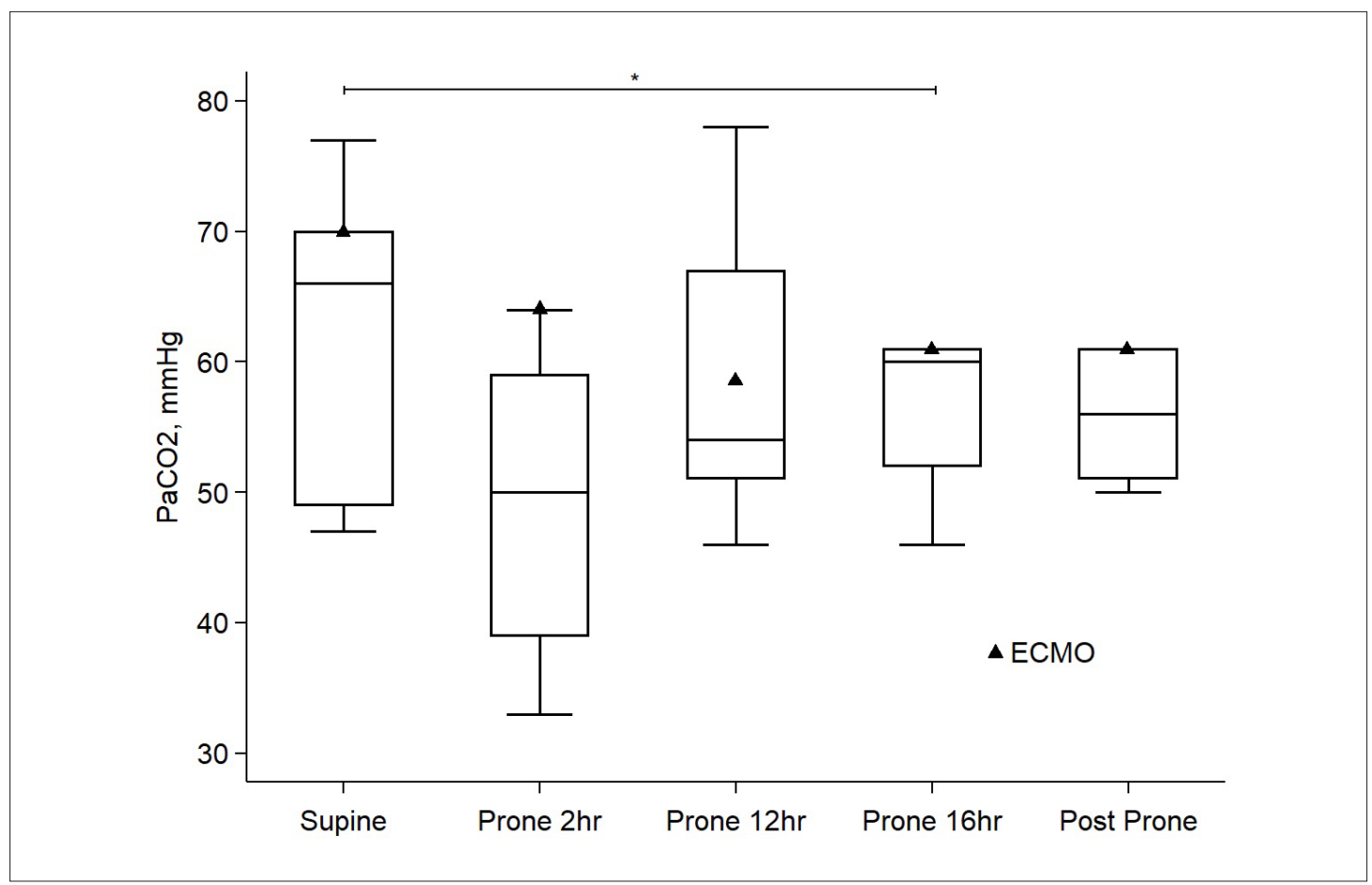

Figure 2: Arterial partial pressure of carbon dioxide ( $\mathrm{PaCO} 2)$ before, during and after prone positioning in patients with COVID-19 acute respiratory distress syndrome.

Triangle sign denotes the patient who did not respond to prone positioning and subsequently required extra-corporeal membrane oxygenation therapy. * $P=0.04$ (z-score -2.032) by Wilcoxon matched-pairs signed-rank test. The rest of between group comparisons were not statistically significant. 


\section{Acknowledgements}

Dr Geak Poh Tan and Dr Rui Min Lee contributed equally to the writing of this manuscript.

\section{REFERENCES}

1. Guerin C, Reignier J, Richard J-C., Beuret P, Gacouin A, Boulain T et al. Prone Positioning in Severe Acute Respiratory Distress Syndrome. N Engl J Med 2013;368:2159-2168.

2. Munshi L, Sorbo LD, Adhikari NKJ, Hodgson CL, Wunsch H, Meade MO et al. Prone Position for Acute Respiratory Distress Syndrome. A Systematic Review and Meta-analysis. Ann Am Thorac Soc 2017;14:S280-S288.

3. Yang X, Yu Y, Xu J, Shu H, Xia J, Liu H et al. Clinical course and outcomes of critically ill patients with SARS-CoV-2 pneumonia in Wuhan, China.Lancet Respir Med 2020;8:475-481.

4. Goh KJ, Choong MC, Cheong EH, Kalimuddin S, Duu Wen S, Phua GC et al. Rapid Progression to Acute Respiratory Distress Syndrome: Review of Current Understanding of Critical Illness from Coronavirus Disease 2019 (COVID-19) Infection. Ann Acad Med Singapore 2020;49:108-18

5. Peek GJ, Mugford M, Tiruvoipati R, Wilson A, Allen E, Thalanany $\mathrm{MM}$ et al. Efficacy and economic assessment of conventional ventilatory support versus extracorporeal membrane oxygenation for severe adult respiratory failure (CESAR): a multicentre randomised controlled trial. Lancet 2009;374:1351-1363.
6. Lim JKB, Qadri SK, Toh TSW, Lin CB, Mok YH, Lee JH. Extracorporeal Membrane Oxygenation for Severe Respiratory Failure during Respiratory Epidemics and Pandemics: A Narrative Review. Ann Acad Med Singapore 2020;49:199-214

Rui Min Lee, ${ }^{1}$ MBBS, Geak Poh $\underline{\text { Tan }},{ }^{2}$ MBChB, Ser Hon Puah, ${ }^{2} M B B S$, Li Min Ling, ${ }^{3} M B B S$, Chiaw Yee Choy, ${ }^{3} M B B S, M R C P(U K)$, Sanjay H Chotirmall, ${ }^{4} M D, P h D, F R C P I$, John Abisheganaden, ${ }^{1,4}$ Jee Jian $\underline{\text { See }},{ }^{1}$ MBBS, Hui Ling Tan, ${ }^{1} M B B S$, Yu-Lin Wong, ${ }^{1} M B B S$

${ }^{1}$ Department of Anaesthesiology, Intensive Care and Pain Medicine, Tan Tock Seng Hospital, Singapore

${ }^{2}$ Department of Respiratory and Critical Care Medicine, Tan Tock Seng Hospital, Singapore

${ }^{3}$ National Centre of Infectious Diseases, Tan Tock Seng Hospital, Singapore ${ }^{4}$ Lee Kong Chian School of Medicine, Nanyang Technological University, Singapore

Address for Correspondence: Dr Rui Min Lee, Department of Anaesthesiology, Intensive Care and Pain Medicine, Tan Tock Seng Hospital, 11 Jalan Tan Tock Seng, Singapore 308433

Email: rui_min_lee@ttsh.com.sg 